\title{
NEW 2H-PYRAZOLO[4,3-c]PYRIDINES: SYNTHESIS, OPTICAL PROPERTIES AND ELUCIDATION OF ANTI-CANCER ACTIVITY
}

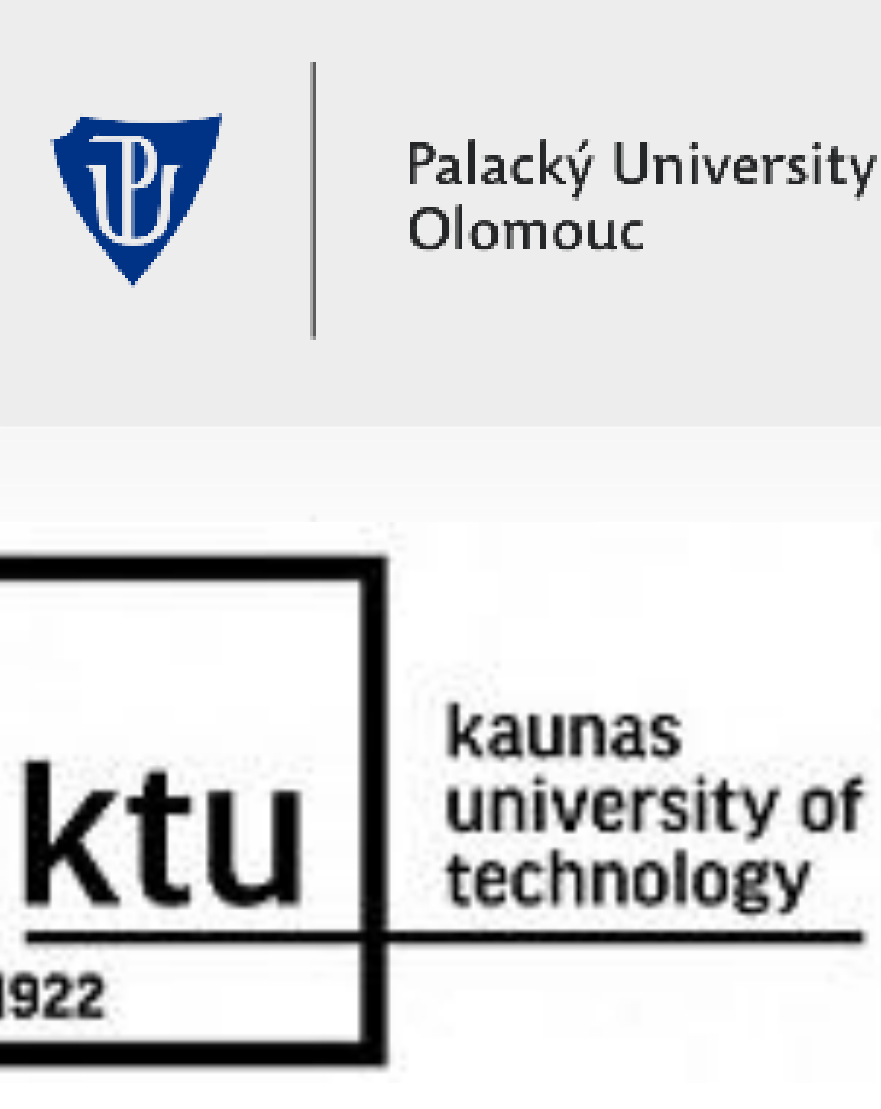

Beatričè Razmienė (1,2)_Eva Rezničková (3), Vaida Dambrauskienė (2), Eglè Arbačiauskienė (2), Martin Kubala (4), Asta Žukauskaitė (3),
Vladimir Kryštof (3), Algirdas Šačkus (1,2)
1) Institute of Synthetic Chemistry, Kaunas University of Technology, K. Baršausko g. 59, LT-51423 Kaunas, Lithuania
2) Department of Organic Chemistry, Kaunas University of Technology, Radvilènu pl. 19, LT-50254, Kaunas, Lithuania
3) Laboratory of Growth Regulators, Institute of Experimental Botany of the Czech Academy of Sciences \& Palacký University, Šlechtitelì 27, CZ-78371 Olomouc,
Czech Republic
4) Department of Experimental Physics, Faculty of Science, Palacký University, 17. Listopadu 12, CZ-77146 Olomouc, Czech Republic

\section{Introduction}

Pyrazole is a common structural unit in many pharmaceuticals and a central axis of numerous ongoing studies devoted to the synthesis and biological evaluation of novel pyrazole moiety-bearing molecules. Annelated pyrazoles are of particular interest as they constitute the core of several well-known drugs, including Sildenafil, Zaleplon and Allopurinol. Among the vast variety of up to now developed biologically active annelated pyrazole derivatives, synthetically demanding $2 \mathrm{H}$-pyrazolo[4,3c]pyridines are, however, relatively understudied. Thus, the aim of this work was to synthesize and evaluate the biological acitivity of novel $2 \mathrm{H}$-pyrazolo[4,3-c]pyridine derivatives.

Firstly, 1-phenyl-3-(2-phenylethynyl)-1 $H$-pyrazole-4carbaldehyde was prepared from 1-phenyl-1 $H$-pyrazol-3ol by consecutive alkylation, formylation and Sonogashira cross-coupling reactions (scheme 1). Then the pyrazolo[4,3-c]pyridine core was obtained via a three step route (scheme 2). Firstly, carbaldehyde $\mathbf{4}$ was converted to alcohols 5 and $\mathbf{6}$ using either Gringnard reagent or reduction conditions and then transformed into azide-alkynes $\mathbf{7}$ and $\mathbf{8}$. The latter were used in electrophilic cyclization reaction to obtain 7-iodo-2,6diphenyl-2H-pyrazolo[4,3-c]pyridines 9 and 10. The library of 2,4,6,7-tetrasubstituted-2H-pyrazolo[4,3c]pyridine derivatives was obtained via palladium catalysed Suzuki-Miyaura cross-coupling reactions.

The newly synthesized compounds were evaluated for their cytotoxicity against two human cancer cell lines: K562 (chronic myeloid leukemia cells) and MCF-7 (breast cancer cells). In general, most tested compounds exhibited moderate cytotoxicity, with $\mathrm{GI}_{50}$ values in the micromolar range (table $\mathbf{1}$ ).

The optical properties of new derivatives were also assessed in THF solutions (table 2).

\section{Optical properties}

\begin{tabular}{|c|c|c|c|}
\hline Compound & $\begin{array}{c}\text { Emision } \boldsymbol{\lambda}_{\text {em }} \\
(\mathbf{n m})\left(\boldsymbol{\lambda}_{\text {ex 350nm }}\right)\end{array}$ & $\begin{array}{c}\text { Stokes } \\
\text { shift } \\
(\mathbf{n m})\end{array}$ & $\begin{array}{c}\text { Quantum } \\
\text { yield } \boldsymbol{\Phi}_{\boldsymbol{f}}(\boldsymbol{\%})\end{array}$ \\
\hline $\mathbf{1 1}$ & 442 & 130 & 18.91 \\
\hline $\mathbf{1 2}$ & 461 & 151 & 71.77 \\
\hline $\mathbf{1 3}$ & 437 & 126 & 53.21 \\
\hline $\mathbf{1 4}$ & 447 & 136 & 26.15 \\
\hline $\mathbf{1 5}$ & 478 & 167 & 56.84 \\
\hline $\mathbf{1 6}$ & 449 & 138 & 17.65 \\
\hline $\mathbf{1 7}$ & 466 & 117 & 72.21 \\
\hline $\mathbf{1 8}$ & 450 & 140 & 48.67 \\
\hline $\mathbf{1 9}$ & 449 & 139 & 30.03 \\
\hline $\mathbf{2 0}$ & 481 & 125 & 62.84 \\
\hline
\end{tabular}

Table 2. Fluorescence parameters in THF.
Synthesis of 1-phenyl-3-(2-phenylethynyl)-1H-pyrazole-4-carbaldehyde

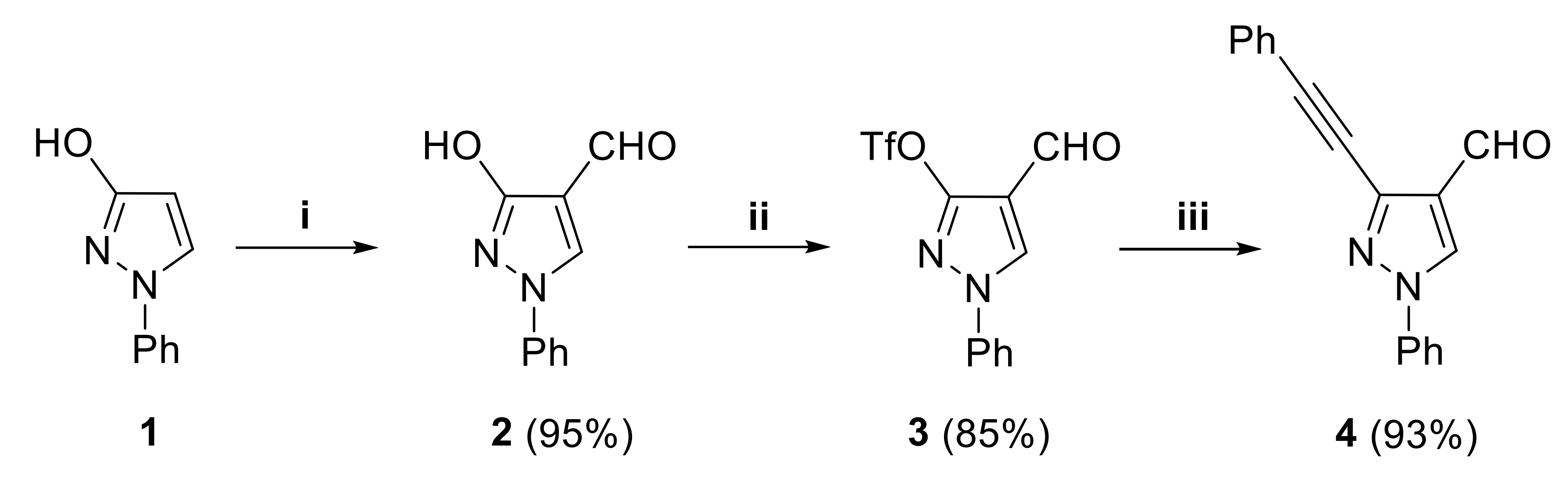

Scheme 1. Reagents: i: (a) $\mathrm{NaH}, \mathrm{BnCl}$; (b) $\mathrm{POCl}_{3}$, DMF; (c) TFA, toluene. ii: $\mathrm{Tf}_{2} \mathrm{O}$, TEA. iii: Phenylacetylene, TEA, CuI, $\mathrm{Pd}\left(\mathrm{PPh}_{3}\right)_{2} \mathrm{Cl}_{2}$.

\section{Synthesis of 2,4,6,7-tetrasubstituted-2H-pyrazolo[4,3-c]pyridines}

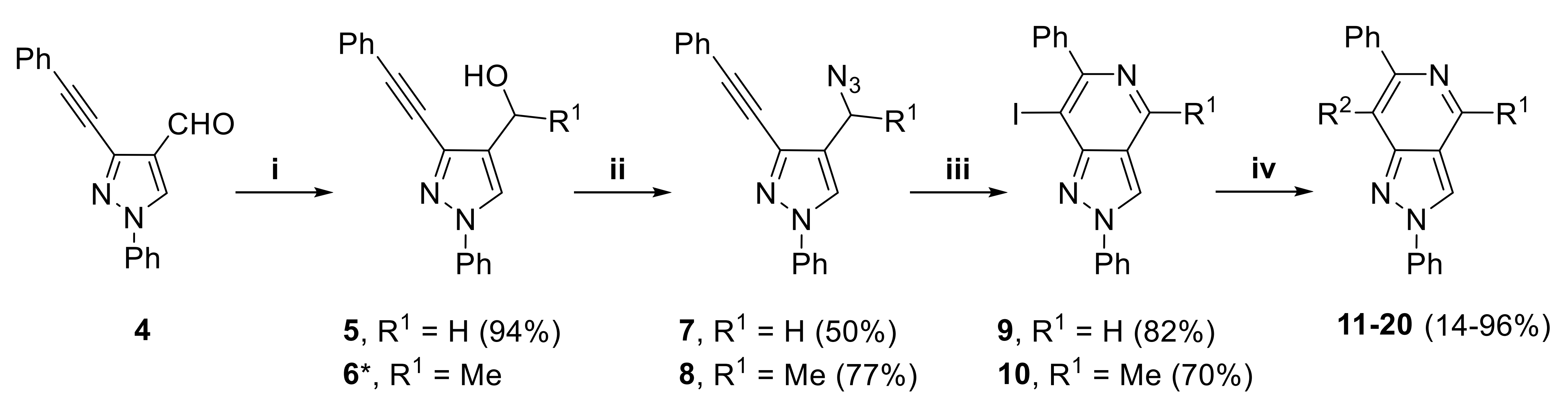

Scheme 2. Reagents: i: $\mathrm{MeMgBr}$ (for 6) or $\mathrm{NaBH}_{4}$ (for 5); ii: $\mathrm{TMSN}_{3}, \mathrm{BF}_{3} \cdot \mathrm{Et}_{2} \mathrm{O}$; iii: $\mathrm{NaHCO}_{3}, \mathrm{I}_{2}$ (for 10) or $\mathrm{K}_{3} \mathrm{PO}_{4} \mathrm{I}_{2}$ (for 9); iv: $\mathrm{R}^{2} \mathrm{~B}(\mathrm{OH})_{2}, \mathrm{Pd}(\mathrm{OAc})_{2}, \mathrm{Cs}_{2} \mathrm{CO}_{3}$. *compound 6 was used in the next step without further purification.

\section{Biological Activity}

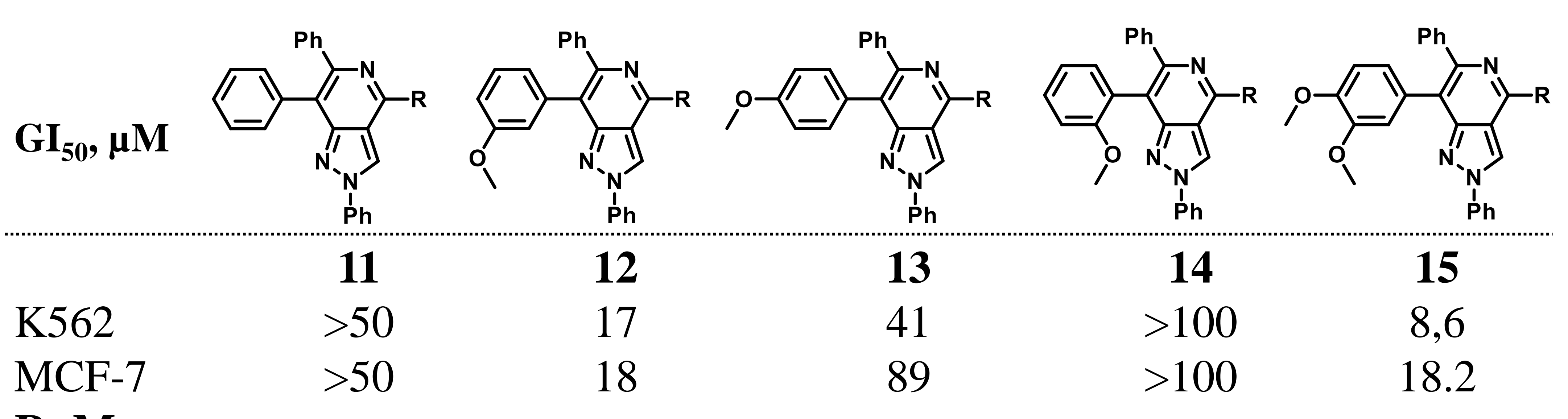

$\mathrm{R}=\mathrm{Me}$

$\begin{array}{lccccc} & \mathbf{1 6} & \mathbf{1 7} & \mathbf{1 8} & \mathbf{1 9} & \mathbf{2 0} \\ \text { K562 } & 10.2 & 2,3 & 4 & 4,5 & 3,9 \\ \text { MCF-7 } & >12.5 & >12.5 & 17.5 & 17.5 & 9.4 \\ \mathbf{R = H} & & & & & \end{array}$

Table 1. In vitro citotoxicity of 2,4,6,7-tetrasubstituted-2H-pyrazolo[4,3-c]pyridines against breast carcinoma MCF-7 and leukemia K562 cell lines.

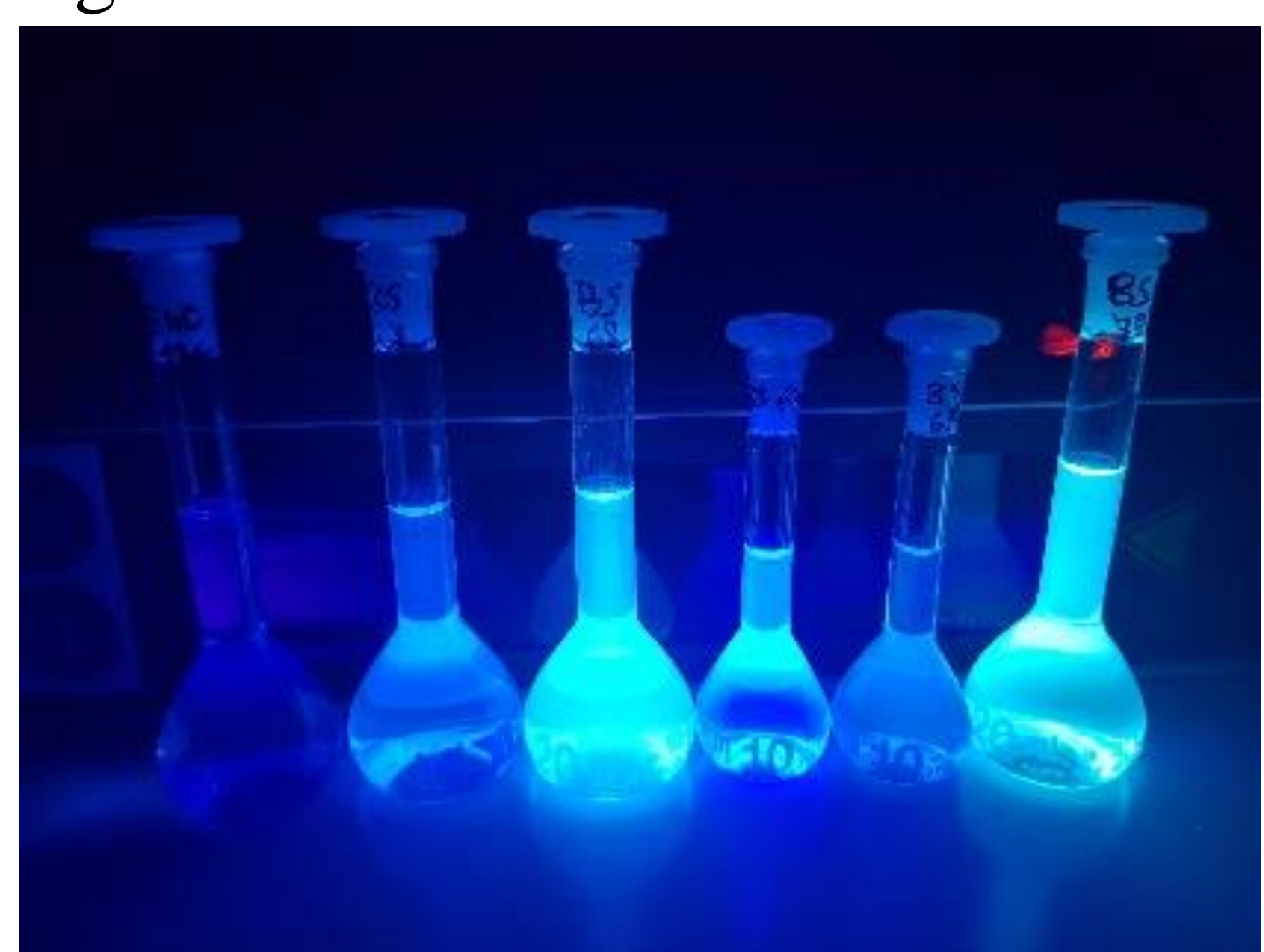

Acknowledgements

This work was supported by the Research Council of Lithuania (LMTLT), agreement No S-MIP-20-60. 